\section{Avaliação do programa de uso da imunoglobulina palivizumabe no Estado de São Paulo, Brasil}

\author{
Assessment of the program for use of the \\ monoclonal antibody palivizumab in \\ São Paulo State, Brazil
}

Evaluación del programa de uso de la inmunoglobulina
palivizumab en el Estado de São Paulo, Brasil
Ivana Regina Gonçalves 1,2

Helio Rubens de Carvalho Nunes 1

Marli Teresinha Cassamassimo Duarte 1

Cristina Maria Garcia de Lima Parada 1

doi: 10.1590/0102-311X00117816

\section{Resumo}

O objetivo deste estudo foi avaliar o programa de uso da imunoglobulina palivizumabe no Estado de São Paulo, Brasil. Adotou-se o referencial de avaliação proposto por Donabedian, e os dados foram discutidos com base nas recomendações da Portaria que regulamenta o uso da palivizumabe em rede nacional e no Manual de Normas e Procedimentos para Vacinação. Foram incluídos os 16 locais de aplicação do estado, bem como 693 crianças/mães inscritas no programa em 2014 (85,1\% da população elegível). Para avaliação da estrutura e processo foram criados escores que permitiram classificar os locais de aplicação em adequado, parcialmente adequado e inadequado, quando havia até 10\%, de 11-20\% e superior a 20\% de desconformidades, respectivamente. Para a avaliação de resultado, buscou-se associação entre falha na tomada da palivizumabe e a necessidade de hospitalização por doença/sintomatologia respiratória, baseando-se em odds ratio pontual e intervalar, com intervalo de $95 \%$ de confiança e valor de p crítico < 0,05. Dos 11 postos de aplicação que tiveram estrutura classificada como adequada, apenas dois apresentaram processo adequado, quatro apresentaram processo inadequado e cinco parcialmente adequados. O risco de hospitalização em UTI por doença/sintomatologia respiratória aumentou em média 30\% a cada falha $(p=0,003 ; O R=$ 1,30; IC95\%: 1,09-1,55). Conclui-se que ter estrutura do programa de uso da imunoglobulina no Estado de São Paulo favorável não resultou, necessariamente, em processo adequado. Em geral, a situação da estrutura foi melhor do que a de processo. Mostrou-se relevante a aplicação de todas as doses da imunoglobulina para a prevenção de internação por doença/sintomatologia respiratória.

Palivizumabe; Imunoglobulinas; Imunização Passiva; Grupos de Risco; Avaliação de Programas e Serviços de Saúde

\author{
Correspondência \\ I. R. Gonçalves \\ Rua Ranimiro Lotufo 593, Botucatu, SP 18606-770, Brasil. \\ ivanagoncalves@fmb.unesp.br \\ 1 Faculdade de Medicina de Botucatu, Universidade Estadual \\ Paulista "Júlio de Mesquita Filho", Botucatu, Brasil. \\ 2 Faculdade Sudoeste Paulista, Avaré, Brasil.
}




\section{Introdução}

A infecção pelo vírus sincicial respiratório (VSR) é a principal causa de mortes infantis no contexto mundial 1,2,3. Esse vírus destaca-se como o mais importante patógeno viral causador da infecção respiratória do trato inferior (IRTI) em lactentes e crianças menores de cinco anos 4 . Segundo a Organização Mundial da Saúde (OMS), o VSR é responsável por cerca de 60 milhões de infecções e 160 mil mortes anuais em todo o mundo 5 .

Ainda que o impacto do VSR sobre a saúde infantil esteja claro, nenhuma vacina segura e eficaz está disponível para a sua prevenção. Diante dessa importante limitação foram desenvolvidos os anticorpos monoclonais dirigidos ao VSR, dos quais o mais favorável é a imunoglobulina palivizumabe (Synagis; Laboratório MedImmune, Gaithersburg, Estados Unidos), aprovada em 1998 para prevenção de doença severa causada pelo VSR em nascidos pré-termo e crianças com doença pulmonar crônica 6 e, posteriormente, às crianças com doença cardíaca congênita 7,8 .

No Brasil, a palivizumabe foi aprovada pela Agência Nacional de Vigilância Sanitária (Anvisa) no ano de 1999, sendo o Estado de São Paulo o primeiro a adotá-la, a partir da Resolução SS no 249 9 , de 13 de julho de 2007. Sua indicação para todo o território nacional foi feita por intermédio da Portaria no 522 10, que apresenta como população-alvo crianças menores de um ano, nascidas prematuras extremas, com idade gestacional igual ou inferior a 28 semanas; e crianças com até dois anos de idade, pneumopatas crônicas ou portadoras de doença cardíaca congênita com repercussão hemodinâmica. Um estudo pioneiro sobre sua eficácia indica que a profilaxia com palivizumabe reduz a necessidade de hospitalização em prematuros 11 .

Nenhuma avaliação sistemática foi realizada sobre o programa de uso dessa imunoglobulina desde o seu início, no âmbito nacional ou estadual. Assim, tem-se por objetivo avaliar o programa de uso da imunoglobulina palivizumabe no Estado de São Paulo.

\section{Método}

Trata-se de estudo inserido no campo da avaliação dos serviços de saúde. Os referenciais de análise adotados foram as políticas públicas de saúde da área, especialmente dois documentos: a Portaria no 522, de 13 de maio 2013 10, que regulamenta o uso da palivizumabe em rede nacional como profilaxia de infecções graves associadas ao VSR, e o Manual de Normas e Procedimentos para Vacinação 12. Como referencial de avaliação de serviços de saúde, adotou-se a proposta de Donabedian ${ }^{13}$, incluindo os componentes estrutura, processo e resultado.

O componente estrutura avalia os recursos existentes para a execução das ações e compreende os recursos físicos, materiais e humanos, as normas e rotinas estabelecidas. A avaliação de processo corresponde às atividades realizadas pelos serviços de saúde, incluindo aspectos técnicos e de relação interpessoal, e a avaliação de resultado é relativa ao efeito que as ações e procedimentos apresentaram sobre a saúde dos usuários ou da população e as relações entre os usuários e os provedores, intermediadas pelas expectativas de ambos 13 .

Os procedimentos para a coleta de dados, realizada entre março e agosto de 2014, incluíram a solicitação de consentimento à Coordenação de Ciência, Tecnologia e Insumos Estratégicos da Secretaria de Estado da Saúde de São Paulo e Programa Estadual de Imunizações; contato telefônico com os responsáveis pelos locais de aplicação, para convite à participação; realização de entrevista telefônica gravada com os gestores destes locais sobre a estrutura e o processo desenvolvido, e entrevistas mensais com as mães das crianças, para avaliação de resultado.

O desenvolvimento do programa depende da identificação das crianças que compõem a população-alvo no período de sazonalidade por qualquer profissional de saúde e encaminhamento para avaliação médica. Confirmando a indicação, o médico atendente deve preencher a Ficha de Solicitação da Imunoglobulina Palivizumabe, encaminhando-a ao local de aplicação mais próximo. Caso o local seja autorizador, sendo a indicação ratificada, é realizado contato com a mãe informando sobre o agendamento da primeira aplicação. Quando o local de aplicação não é autorizador, a ficha de solicitação é encaminhada, via Grupo de Vigilância Epidemiológica, ao Programa Estadual de Imunizações que, confirmando a indicação, informa o local de aplicação de referência e este realiza o contato com a mãe 
para agendamento da aplicação. Em ambos os casos, o agendamento periódico e a aplicação da imunoglobulina constituem responsabilidade dos locais de aplicação, que estabelecem os números de doses que cada criança deverá receber, a depender da data de autorização e do período sazonal. Anualmente, porém, o programa é iniciado após anuência da Secretaria de Estado da Saúde.

Não houve recusa dos gestores, sendo incluídos todos os locais de aplicação paulistas, divididos em Centros de Referência de Imunobiológicos Especiais (CRIE) ABC, Botucatu, Campinas, Ribeirão Preto e Universidade Federal de São Paulo (Unifesp), que também participam da logística e aplicação dos demais imunobiológicos especiais fornecidos pelo Programa Nacional de Imunizações, além dos postos da Casa de Saúde de Araçatuba, Conjunto Hospitalar de Sorocaba, Hospital de Base de São José do Rio Preto, Hospital das Clínicas de Marília, Hospital Estadual Bauru, Hospital Estadual Guilherme Álvaro, Hospital Estadual de Presidente Prudente, Hospital Maternidade Interlagos, Hospital Maternidade Leonor Mendes de Barros, Instituto da Criança de São Paulo e Posto de Assistência Médico Odontológico de Taubaté, dos quais apenas o CRIE de Ribeirão Preto e o Hospital Estadual de Presidente Prudente são autorizadores. Destaca-se que o CRIE de Botucatu foi excluído da análise de estrutura e processo por tratar-se do local de trabalho da primeira autora desta pesquisa. Os dados do referido local, no entanto, integraram a análise de falhas de aplicação da imunoglobulina palivizumabe.

Foram critérios de inclusão das crianças: participar do programa no ano de 2014, ter menos de 24 meses no período sazonal e residir no Estado de São Paulo durante a coleta de dados. Os critérios de exclusão foram: óbito materno ou da criança no período de sazonalidade, dificuldade de comunicação materna e a criança residir longe da mãe.

Considerando todo o Estado de São Paulo, 872 crianças que atendiam à Portaria no 522 10, foram incluídas no programa no mês de seu início. No entanto, a população elegível para este estudo foi composta por 814 crianças, uma vez que 58 não atendiam aos critérios de inclusão: 29 completariam 24 meses no período de sazonalidade; quatro haviam perdido a mãe por óbito; sete tinham mãe com dificuldade de comunicação; oito residiam fora do Estado de São Paulo; seis viviam em abrigo e quatro crianças faleceram antes do término do período de sazonalidade. Foram perdidas 121 crianças: 29 por recusa materna e 92 mães não foram localizadas após sete ligações telefônicas em pelo menos dois horários e dias distintos. Assim, a amostra final foi constituída por 693 crianças $(85,1 \%$ da população elegível): 189 prematuras, 133 cardiopatas, seis pneumopatas, 147 prematuras e pneumopatas, 130 prematuras e cardiopatas, 13 pneumopatas e cardiopatas e 75 prematuras, pneumopatas e cardiopatas.

A coleta de dados incluiu variáveis sociodemográficas para a caracterização materna: cor (branca, não branca); idade em anos (até 19, 20-34, 35 ou mais); anos de aprovação escolar (até 7, 8-10, 11 ou mais); trabalho remunerado (sim, não) e companheiro (sim, não); e da criança: idade gestacional ao nascer em semanas (até 27, 28 ou mais); idade em meses na primeira dose da imunoglobulina (até 5, 6-12, 13-24); recebeu palivizumabe no ano anterior (sim, não); história de doença com indicação cirúrgica (sim, não); história de doença clínica (sim, não), histórico de prematuridade (sim, não).

Com relação às variáveis relativas à estrutura dos locais de aplicação, foram incluídos dados dos recursos materiais (sim, não), considerando-se: equipamento para a conservação do imunobiológico para o dia de trabalho; equipamento para estoque do imunobiológico; termômetro de máxima e mínima temperaturas e mapa para controle de temperatura; balança; medicamentos e material para uso em caso de choque anafilático; impressos preconizados para rotina de trabalho na sala de vacina; seringas e agulhas de diferentes calibres estocadas adequadamente; caixa rígida para descarte de resíduos perfuro-cortantes; compressas de álcool a 70\%; disponibilidade do imunobiológico para todo o período de sazonalidade e existência de protocolo de uso da palivizumabe, disponilizado de forma impressa. Excetuando-se os dois últimos itens, incluídos pela relevância, os demais constam das políticas oficiais, referências deste estudo 10,12 .

Incluíram-se dados relativos aos recursos físicos (sim, não): sala de aplicação climatizada, exclusiva para aplicação de imunobiológicos; conservação adequada; ausência de decoração; piso adequado; proteção da luz solar direta; mobiliário bem distribuído; fácil acesso da população; iluminação adequada; existência de pia para higienização das mãos; bancada higienizável; tamanho mínimo de $6 \mathrm{~m}^{2}$; parede clara, impermeável e higienizável e limpeza adequada.

Sobre os recursos humanos, foram investigadas (sim, não) com base nas políticas oficiais 10,12: presença de enfermeiro ou enfermeiro supervisor; capacitação do enfermeiro e/ou do técnico/auxiliar 
de enfermagem para administração da imunoglobulina; equipe treinada para atendimento de reações adversas; presença de profissional técnico-administrativo para agendamento, recepção dos clientes e registro de informações; equipe constituída por médico e enfermeiro/técnico de enfermagem; presença de médico durante a aplicação e capacitação inicial da enfermeira/equipe pela Secretaria de Estado da Saúde ou Grupo de Vigilância Epidemiológica (sendo este último item proposto especificamente para este estudo, por sua relevância).

Variáveis relativas ao processo (sim, não) também constam dos documentos adotados como referência 10,12: preenchimento do mapa de controle da temperatura no mínimo duas vezes ao dia; realização de limpeza quinzenal; aplicação da imunoglobulina em caso de hospitalização da criança; supervisão do profissional médico durante as aplicações; reconstituição e conservação da imunoglobulina em temperatura entre $+2 \mathrm{e}+8^{\circ} \mathrm{C}$; primeira aplicação no mês anterior ao período de sazonalidade; busca ativa de crianças faltosas; administrações superiores a 1,0mL, divididas e aplicadas em grupos musculares distintos; registro da aplicação no cartão da criança, contendo o lote e a data da próxima aplicação e realização de orientação verbal sobre a imunoglobulina para a mãe (esta última proposta para esta investigação por ser considerada relevante ao bom desenvolvimento do projeto).

A avaliação de resultado foi realizada baseando-se nas crianças inscritas no programa. Considerou-se variável de desfecho a ocorrência de hospitalização (sim, não) em UTI por doença/sintomas respiratórios, observando-se o grupo que seguiu o protocolo e o grupo que não o seguiu (falha). Considerou-se ter havido falha quando a aplicação de uma dose foi realizada com intervalo superior a trinta dias da dose anterior e/ou na ausência da aplicação de qualquer dose do esquema proposto e/ou quando, apesar de terem sido aplicadas as cinco doses, não tiver sido atendido ao preconizado: início um mês antes do período de sazonalidade do VSR (no Estado de São Paulo, no mês de março) 10. Assim, considerando-se o grupo que não seguiu o protocolo, ocorreram 599 falhas por não terem sido aplicadas as cinco doses e, destas, 588 por ausência de aplicação no mês anterior à sazonalidade; outras 78 falhas decorreram da aplicação da imunoglobulina com intervalo superior a 30 dias, totalizando 677 crianças que não seguiram o protocolo.

A avaliação de resultado considerou três hipóteses: (1) o risco de hospitalização em UTI por doença/sintomas respiratórios é maior entre crianças que falharam em pelo menos uma dose da imunoglobulina em relação às crianças que tomaram todas as doses; (2) o risco de hospitalização em UTI por doença/sintomas respiratórios aumenta com o aumento do número de falhas na tomada da imunoglobulina; e (3) orisco de hospitalização em UTI por doença/sintomas respiratórios está associado à falha na tomada da palivizumabe no mês anterior à observação do desfecho.

A análise de estrutura e processo foi realizada descritivamente. Para tanto, criou-se escore de estrutura, considerando-se os 13 itens relativos aos recursos físicos, 11 sobre recursos materiais e seis itens de recursos humanos, sendo atribuído um ponto para cada item em desconformidade. A estrutura foi classificada como adequada quando houve entre um e três itens em desconformidade (até 10\% do total de itens considerados); parcialmente adequada quando quatro a seis itens estavam em desconformidade (até 20\%); e inadequada quando sete ou mais itens estavam em desconformidade (acima de 20\%).

O escore de processo considerou dez variáveis, sendo adequado quando houve uma desconformidade (10\%); parcialmente adequado na vigência de duas desconformidades (20\%); e havendo três ou mais desconformidades, considerou-se processo inadequado (acima de $20 \%$ ).

A análise de resultado foi realizada por estimativa pontual e intervalar da chance de hospitalização em UTI por doença ou sintoma respiratório no período de sazonalidade, entre março e agosto de 2014. A medida de associação utilizada foi o odds ratio (OR) pontual, com intervalo de $95 \%$ de confiança (IC95\%). Associações foram consideradas significativas se $\mathrm{p}<0,05$. A análise foi realizada com o software IBM SPSS 21 (IBM Corp., Armonk, Estados Unidos).

Este estudo foi aprovado pelo Comitê de Ética em Pesquisa (no 551.293) e seguiu as normas preconizadas pela Resolução no 466/2012, do Conselho Nacional de Saúde. Para respeitar o sigilo, os locais de aplicação foram representados pela letra "C", no caso dos CRIE, e letra "P" no caso dos postos de aplicação, seguido do número correspondente. 


\section{Resultados}

A caracterização das mães evidencia que eram predominantemente da cor branca $(64,6 \%)$, na faixa etária entre 20-34 anos (65,2\%), com 11 ou mais anos de estudos (80,5\%), trabalho remunerado $(52,4 \%)$ e companheiro $(92,2 \%)$. Quanto às crianças, a maioria tinha entre 6-12 meses na primeira dose (56\%), $26,1 \%$ haviam recebido palivizumabe no ano anterior à pesquisa e $317(45,7 \%)$ possuíam histórico de doença cirúrgica, sendo 187 casos de cardiopatia, 22 de pneumopatia, três de cardiopatia e pneumopatia e 105 de outras patologias cirúrgicas (dados não apresentados em tabela).

Todas as crianças tinham história de doença clínica, sendo os sistemas mais acometidos o gastrointestinal ( 77 crianças) e o nervoso ( 55 casos). A taxa de prematuridade foi de $84 \%$, sendo que $53,1 \%$ tinham nascido com idade gestacional igual ou superior a 28 semanas (dados não apresentados em tabela).

Aspectos da estrutura física em desconformidade eram relativos à: climatização (8/15); sala exclusiva para o procedimento (4/15); sala em condições ideais de conservação (2/15); presença de decoração (1/15); piso resistente e antiderrapante (1/15); proteção contra luz solar direta (1/15); e mobiliário com distribuição funcional (1/15). Entre os recursos materiais: medicamentos e materiais para uso em caso de choque anafilático (5/15), protocolo de uso da palivizumabe impresso e disponível aos funcionários (3/15), compressas de álcool 70\% (1/15) e disponibilidade da imunoglobulina em todo o período de sazonalidade (1/15) (Tabela 1$)$.

\section{Tabela 1}

Conformidades e desconformidades relativas aos recursos físicos e materiais dos locais de aplicação da imunoglobulina palivizumabe e escore gerado. Estado de São Paulo, Brasil, 2014.

\begin{tabular}{|c|c|c|c|c|c|c|c|c|c|c|c|c|c|c|c|}
\hline Recursos & C1 & C2 & C3 & C4 & P1 & $\mathbf{P 2}$ & P3 & P4 & P5 & P6 & P7 & P8 & P9 & P10 & P11 \\
\hline \multicolumn{16}{|l|}{ Sala de aplicação } \\
\hline Climatizada & $x$ & $x$ & 0 & $x$ & 0 & $x$ & $x$ & $x$ & 0 & 0 & 0 & 0 & 0 & $x$ & $x$ \\
\hline Exclusiva & 0 & 0 & $X$ & 0 & $x$ & 0 & 0 & $x$ & 0 & 0 & $X$ & 0 & 0 & 0 & 0 \\
\hline Bem conservada & 0 & 0 & 0 & 0 & 0 & 0 & 0 & 0 & 0 & $x$ & 0 & 0 & $\mathrm{X}$ & 0 & 0 \\
\hline Sem decoração & 0 & 0 & 0 & 0 & 0 & 0 & 0 & 0 & 0 & 0 & 0 & 0 & $x$ & 0 & 0 \\
\hline Piso adequado & 0 & 0 & 0 & 0 & 0 & 0 & 0 & 0 & 0 & 0 & 0 & 0 & 0 & 0 & $x$ \\
\hline Protegida da luz solar & 0 & 0 & 0 & 0 & 0 & 0 & 0 & 0 & 0 & 0 & 0 & 0 & 0 & 0 & $x$ \\
\hline Mobíliário distribuído & 0 & 0 & 0 & 0 & 0 & 0 & 0 & 0 & 0 & $x$ & 0 & 0 & 0 & 0 & 0 \\
\hline Fácil acesso & 0 & 0 & 0 & 0 & 0 & 0 & 0 & 0 & 0 & 0 & 0 & 0 & 0 & 0 & 0 \\
\hline Iluminação adequada & 0 & 0 & 0 & 0 & 0 & 0 & 0 & 0 & 0 & 0 & 0 & 0 & 0 & 0 & 0 \\
\hline Pia/Bancada higienizável & 0 & 0 & 0 & 0 & 0 & 0 & 0 & 0 & 0 & 0 & 0 & 0 & 0 & 0 & 0 \\
\hline Tamanho $\geq 6 \mathrm{~m}^{2}$ & 0 & 0 & 0 & 0 & 0 & 0 & 0 & 0 & 0 & 0 & 0 & 0 & 0 & 0 & 0 \\
\hline Parede impermeável, clara e higienizável & 0 & 0 & 0 & 0 & 0 & 0 & 0 & 0 & 0 & 0 & 0 & 0 & 0 & 0 & 0 \\
\hline Limpeza adequada & 0 & 0 & 0 & 0 & 0 & 0 & 0 & 0 & 0 & 0 & 0 & 0 & 0 & 0 & 0 \\
\hline \multicolumn{16}{|l|}{ Materiais disponíveis } \\
\hline Caixa/Equipamento para dia de trabalho & 0 & 0 & 0 & 0 & 0 & 0 & 0 & 0 & 0 & 0 & 0 & 0 & 0 & 0 & 0 \\
\hline Equipamento para estoque imunobiológico & 0 & 0 & 0 & 0 & 0 & 0 & 0 & 0 & 0 & 0 & 0 & 0 & 0 & 0 & 0 \\
\hline Termômetro/Mapa temperatura & 0 & 0 & 0 & 0 & 0 & 0 & 0 & 0 & 0 & 0 & 0 & 0 & 0 & 0 & 0 \\
\hline Balança infantil & 0 & 0 & 0 & 0 & 0 & 0 & 0 & 0 & 0 & 0 & 0 & 0 & 0 & 0 & 0 \\
\hline Medicamentos/Material para choque & $x$ & 0 & $x$ & 0 & 0 & $x$ & 0 & $x$ & 0 & 0 & 0 & 0 & 0 & 0 & $x$ \\
\hline Protocolo palivizumabe impresso & 0 & 0 & $x$ & 0 & 0 & 0 & 0 & 0 & 0 & 0 & 0 & 0 & 0 & $x$ & $x$ \\
\hline Compressa (álcool a 70\%) & 0 & 0 & 0 & 0 & 0 & 0 & 0 & 0 & 0 & $x$ & 0 & 0 & 0 & 0 & 0 \\
\hline Imunoglobulina sempre disponível & 0 & 0 & 0 & 0 & 0 & 0 & 0 & 0 & $x$ & 0 & 0 & 0 & 0 & 0 & 0 \\
\hline Impressos preconizados & 0 & 0 & 0 & 0 & 0 & 0 & 0 & 0 & 0 & 0 & 0 & 0 & 0 & 0 & 0 \\
\hline Seringas/Agulhas estocadas adequadamente & 0 & 0 & 0 & 0 & 0 & 0 & 0 & 0 & 0 & 0 & 0 & 0 & 0 & 0 & 0 \\
\hline Caixa para perfuro-cortante & 0 & 0 & 0 & 0 & 0 & 0 & 0 & 0 & 0 & 0 & 0 & 0 & 0 & 0 & 0 \\
\hline Escore & 2 & 1 & 3 & 1 & 1 & 2 & 1 & 3 & 1 & 3 & 1 & 0 & 2 & 2 & 5 \\
\hline
\end{tabular}

0: em conformidade; $\mathrm{X}$ : em desconformidade. 
Entre as desconformidades dos recursos humanos estavam: ausência de profissional técnico-administrativo para agendamento, recepção e registro de informações nos locais de aplicação (5/15); enfermeiro supervisor/enfermeiro nos locais de aplicação (1/15); médico no local de aplicação durante a aplicação (3/15); capacitação inicial das equipes para as atividades do programa pela Secretaria de Estado da Saúde ou Grupo de Vigilância Estadual (7/15); e capacitação da equipe para atendimento a reações adversas (1/15) (Tabela 2$)$.

Relativo aos recursos humanos, considerando-se os escores propostos, a estrutura final de todos os CRIE (C1 a C4) foi considerada adequada, quatro postos de aplicação (P1, P4, P6 e P11) foram classificados como parcialmente adequados. Os demais eram adequados (dados não apresentados em tabela).

Quanto ao processo, as desconformidades dizem respeito à aplicação do imunobiológico em caso de hospitalização da criança (11/15), supervisão do profissional médico durante as aplicações (10/15), conservação do imunobiológico após reconstituição entre +2 e $+8^{\circ} \mathrm{C}(10 / 15)$, primeira aplicação no mês anterior à sazonalidade (5/15) e busca ativa de crianças faltosas (1/15) (Tabela 2).

Considerando-se o escore final de processo, três CRIE (C2, C3 e C4) foram considerados parcialmente adequados e um (C1) inadequado; três postos (P4, P7 e P9) tiveram seu processo considerado adequado, dois (P3 e P10) parcialmente adequados e seis (P1, P2, P5, P6, P8 e P11) inadequados (Tabela 2).

Apenas dois locais de aplicação (P7 e P9) apresentaram estrutura e processo considerados adequados. Apesar de terem estrutura classificada como adequada, nove locais registraram processo parcialmente adequado (C2, C3, C4, P3 e P10) ou inadequado (C1, P2, P5 e P8) (Tabela 3).

Em todos os locais de aplicação houve pelo menos uma falha, sendo que o maior número ocorreu no C3, representando 17\% (118/693) do total de crianças. Quatro locais de aplicação (C3, C4, P9 e P10)

Tabela 2

Conformidades e desconformidades relativas aos recursos humanos e processos desenvolvidos nos locais de aplicação da imunoglobulina palivizumabe no mês de início da aplicação, com os respectivos escores de desconformidades encontradas. Estado de São Paulo, Brasil, 2014.

$\begin{array}{lllllllllllllll}\text { C1 } & \text { C2 } & \text { C3 } & \text { C4 } & \text { P1 } & \text { P2 } & \text { P3 } & \text { P4 } & \text { P5 } & \text { P6 } & \text { P7 } & \text { P8 } & \text { P9 } & \text { P10 } & \text { P11 }\end{array}$

\begin{tabular}{|c|c|c|c|c|c|c|c|c|c|c|c|c|c|c|c|}
\hline \multicolumn{16}{|l|}{ Recursos } \\
\hline \multicolumn{16}{|l|}{ Constituição da equipe } \\
\hline Profissional técnico-administrativo & 0 & $x$ & 0 & 0 & 0 & 0 & 0 & $x$ & 0 & $x$ & 0 & $x$ & 0 & 0 & $x$ \\
\hline Enfermeiro supervisor/Enfermeiro & 0 & 0 & 0 & 0 & $x$ & 0 & 0 & 0 & 0 & 0 & 0 & 0 & 0 & 0 & 0 \\
\hline Médico, enfermeiro/Técnico de enfermagem & 0 & 0 & 0 & 0 & 0 & 0 & 0 & 0 & 0 & 0 & 0 & $x$ & 0 & 0 & 0 \\
\hline Médico durante aplicação & 0 & 0 & 0 & 0 & $x$ & 0 & $x$ & 0 & 0 & $x$ & 0 & 0 & 0 & 0 & 0 \\
\hline \multicolumn{16}{|l|}{ Capacitação profissional } \\
\hline Enfermeiro/Equipe com capacitação inicial & 0 & 0 & 0 & $x$ & $x$ & 0 & $x$ & $x$ & 0 & $x$ & 0 & $x$ & $x$ & 0 & 0 \\
\hline Atendimento a reações adversas & 0 & 0 & 0 & 0 & $x$ & 0 & 0 & 0 & 0 & 0 & 0 & 0 & 0 & 0 & 0 \\
\hline Escore recursos & 0 & 1 & 0 & 1 & 4 & 0 & 2 & 2 & 0 & 3 & 0 & 2 & 1 & 0 & 1 \\
\hline \multicolumn{16}{|l|}{ Processo } \\
\hline Aplica na hospitalização & $x$ & $x$ & 0 & $x$ & $x$ & $x$ & 0 & 0 & $x$ & $x$ & $x$ & $x$ & 0 & $x$ & $x$ \\
\hline Supervisão do médico & $x$ & 0 & $x$ & 0 & $x$ & $x$ & $x$ & 0 & $x$ & $x$ & 0 & $x$ & 0 & $x$ & $x$ \\
\hline Conservação: $+2 \mathrm{e}+8^{\circ} \mathrm{C}$ & $x$ & $x$ & $x$ & $x$ & $x$ & $x$ & $x$ & 0 & $x$ & $x$ & 0 & $x$ & 0 & 0 & 0 \\
\hline Primeira aplicação um mês antes da sazonalidade & $x$ & 0 & 0 & 0 & 0 & $x$ & 0 & $x$ & $X$ & 0 & 0 & 0 & 0 & 0 & $x$ \\
\hline Busca ativa de faltosos & $x$ & 0 & 0 & 0 & 0 & 0 & 0 & 0 & 0 & 0 & 0 & 0 & 0 & 0 & 0 \\
\hline Divisão de doses $>1,0 \mathrm{~mL}$ & 0 & 0 & 0 & 0 & 0 & 0 & 0 & 0 & 0 & 0 & 0 & 0 & 0 & 0 & 0 \\
\hline Controle de temperatura $\geq 2$ vezes/dia & 0 & 0 & 0 & 0 & 0 & 0 & 0 & 0 & 0 & 0 & 0 & 0 & 0 & 0 & 0 \\
\hline Registro do lote e retorno & 0 & 0 & 0 & 0 & 0 & 0 & 0 & 0 & 0 & 0 & 0 & 0 & 0 & 0 & 0 \\
\hline Limpeza quinzenal da sala & 0 & 0 & 0 & 0 & 0 & 0 & 0 & 0 & 0 & 0 & 0 & 0 & 0 & 0 & 0 \\
\hline Orientação sobre o imunobiológico às mães & 0 & 0 & 0 & 0 & 0 & 0 & 0 & 0 & 0 & 0 & 0 & 0 & 0 & 0 & 0 \\
\hline Escore processo & 5 & 2 & 2 & 2 & 3 & 4 & 2 & 1 & 4 & 3 & 1 & 3 & 0 & 2 & 3 \\
\hline
\end{tabular}

0: em conformidade; $X$ : em desconformidade. 
Tabela 3

Classificação da estrutura e processo dos locais de aplicação com base no escore proposto neste estudo. Estado de São Paulo, Brasil, 2014.

\begin{tabular}{lcc}
\hline Local & Estrutura & Processo \\
\hline C1 & Adequada & Inadequado \\
C2 & Adequada & Parcialmente adequado \\
C3 & Adequada & Parcialmente adequado \\
C4 & Adequada & Parcialmente adequado \\
P1 & Parcialmente adequada & Inadequado \\
P2 & Adequada & Inadequado \\
P3 & Adequada & Parcialmente adequado \\
P4 & Parcialmente adequada & Adequado \\
P5 & Adequada & Inadequado \\
P6 & Parcialmente adequada & Inadequado \\
P7 & Adequada & Adequado \\
P8 & Adequada & Inadequado \\
P9 & Adequada & Adequado \\
P10 & Adequada & Parcialmente adequado \\
P11 & Parcialmente adequada & Inadequado \\
\hline
\end{tabular}

concentraram 57,6\% (390/677) de todas as falhas dos 16 locais de aplicação. Considerando-se o maior número de falhas (quatro ou cinco), destacou-se o P11: de 21 crianças atendidas, 14 tiveram quatro falhas (66,7\%). Para 16 (2,3\%) crianças atendidas não houve falha de aplicação de dose (Tabela 4).

Ressalta-se que 84,8\% (588/693) das crianças não receberam a imunoglobulina no mês anterior à sazonalidade (Tabela 5). Dessas, $184(31,3 \%)$ receberam as quatro demais doses corretamente (dado não apresentado em tabela).

Com relação ao resultado do uso da imunoglobulina palivizumabe, observou-se que o risco de hospitalização em UTI por doença/sintomatologia respiratória não diferiu quando consideradas as crianças que falharam na tomada de pelo menos uma dose, comparadas às crianças que tomaram todas as doses (Tabela 5).

Com o aumento na quantidade de falhas, houve aumento na hospitalização em UTI por doença/ sintomatologia respiratória, sendo que a cada falha a chance de hospitalização aumentava em $30 \%$ $(\mathrm{p}=$ 0,003; OR = 1,30; IC95\%: 1,09-1,55). Nos meses de março, abril e junho, a ocorrência de falha no recebimento da imunoglobulina esteve associada à hospitalização da criança em UTI por doença/ sintomatologia respiratória no mês subsequente, respectivamente em abril, maio e julho (Tabela 5).

\section{Discussão}

No ano de 2014, em todo o Estado de São Paulo nasceram, em média mensal, 292 crianças prematuras extremas, com idade gestacional igual ou inferior a 27 semanas (Departamento de Informática do SUS. Nascidos vivos - São Paulo, 2014. http://tabnet.datasus.gov.br/cgi/tabcgi.exe?sinasc/cnv/nvSP. def, acessado em12/Mar/2015). Assim, mesmo considerando apenas esse critério, pode-se inferir ser baixa a cobertura do programa, visto que apenas 872 crianças foram nele incluídas no mês do seu início neste mesmo ano, sendo importante a divulgação das evidências relacionadas aos benefícios do uso da imunoglobulina e formas de acesso ao programa junto a profissionais e usuários dos serviços de saúde materno infantis no estado. Outra importante fragilidade observada está relacionada ao grande número de falhas no recebimento da imunoglobulina.

Quanto às variáveis selecionadas para este estudo, evidenciou-se que embora ainda possa ser melhorada, a estrutura dos locais de aplicação é, em geral, adequada, já o processo desenvolvido 
Tabela 4

Número de falhas de acordo com o local de aplicação da imunoglobulina palivizumabe. Estado de São Paulo, Brasil, 2014.

\begin{tabular}{lccccccccccccccccc} 
Número de falhas & $\mathbf{C 1}$ & $\mathbf{C 2}$ & $\mathbf{C 3}$ & $\mathbf{C 4}$ & $\mathbf{C 5}$ & $\mathbf{P 1}$ & $\mathbf{P 2}$ & $\mathbf{P 3}$ & $\mathbf{P 4}$ & $\mathbf{P 5}$ & $\mathbf{P 6}$ & $\mathbf{P 7}$ & $\mathbf{P 8}$ & $\mathbf{P 9}$ & $\mathbf{P 1 0}$ & $\mathbf{P 1 1}$ & Total \\
\hline 0 & 0 & 4 & 3 & 3 & 0 & 0 & 0 & 0 & 0 & 0 & 1 & 0 & 3 & 2 & 0 & 0 & 16 \\
1 & 9 & 29 & 51 & 51 & 0 & 0 & 22 & 5 & 1 & 1 & 4 & 4 & 4 & 49 & 9 & 0 & 239 \\
2 & 3 & 14 & 32 & 18 & 4 & 1 & 6 & 10 & 1 & 2 & 3 & 4 & 7 & 23 & 36 & 0 & 164 \\
3 & 33 & 27 & 32 & 16 & 6 & 0 & 1 & 16 & 2 & 3 & 1 & 5 & 12 & 13 & 28 & 7 & 202 \\
4 & 8 & 5 & 2 & 2 & 0 & 0 & 0 & 4 & 2 & 1 & 0 & 2 & 1 & 2 & 21 & 14 & 64 \\
5 & 1 & 0 & 1 & 1 & 0 & 0 & 0 & 0 & 0 & 0 & 0 & 2 & 0 & 2 & 1 & 0 & 8 \\
Total de falhas & 54 & 75 & 118 & 88 & 10 & 1 & 29 & 35 & 6 & 7 & 8 & 17 & 24 & 89 & 95 & 21 & 677 \\
Total de crianças & 54 & 79 & 121 & 91 & 10 & 1 & 29 & 35 & 6 & 7 & 9 & 17 & 27 & 91 & 95 & 21 & 693 \\
\hline
\end{tabular}

\section{Tabela 5}

Hospitalização das crianças inscritas no programa de uso da imunoglobulina em UTI por doença/sintoma respiratório ( $N=693)$, considerando desconformidades do programa. Estado de São Paulo, Brasil, 2014.

\begin{tabular}{|c|c|c|c|c|}
\hline Desconformidades & $\begin{array}{c}\text { Total } \\
\mathbf{n}\end{array}$ & $\begin{array}{c}\text { Hospitalizações } \\
\text { n (\%) }\end{array}$ & OR (IC95\%) & Valor de p \\
\hline Falha de pelo menos uma dose & & & $0,96(0,27-342)$ & 0,952 \\
\hline Não & 16 & $3(18,8)$ & & \\
\hline Sim & 677 & $123(18,2)$ & & \\
\hline Quantidade de falhas & & & $1,30(1,09-1,55)$ & 0,003 \\
\hline 0 & 16 & $3(18,8)$ & & \\
\hline 1 & 239 & $32(13,4)$ & & \\
\hline 2 & 164 & $29(17,7)$ & & \\
\hline 3 & 202 & $42(20,8)$ & & \\
\hline 4 & 64 & $16(25,0)$ & & \\
\hline 5 & 8 & $4(50,0)$ & & \\
\hline \multicolumn{5}{|l|}{ Mês de ocorrência da falha * } \\
\hline Março & & & $8,0(1,0-58,7)$ & 0,041 \\
\hline Não & 105 & $1(1,0)$ & & \\
\hline Sim & 588 & $42(7,0)$ & & \\
\hline Abril & & & $2,0(1,04-3,8)$ & 0,038 \\
\hline Não & 537 & $27(5,0)$ & & \\
\hline Sim & 156 & $15(9,6)$ & & \\
\hline Maio & & & $1,5(0,8-2,7)$ & 0,179 \\
\hline Não & 455 & $26(5,7)$ & & \\
\hline Sim & 238 & $20(8,4)$ & & \\
\hline Junho & & & $2,5(1,0-5,76)$ & 0,030 \\
\hline Não & 400 & $9(2,3)$ & & \\
\hline Sim & 293 & $16(5,5)$ & & \\
\hline Julho & & & $1,3(0,6-3,0)$ & 0,428 \\
\hline Não & 499 & $19(3,8)$ & & \\
\hline Sim & 194 & $10(5,2)$ & & \\
\hline
\end{tabular}

IC95\%: intervalo de 95\% de confiança; OR: odds ratio.

* Mês da falha, com hospitalização no mês subsequente. 
requer melhorias em vários aspectos. Quanto ao resultado, na medida em que aumentaram as falhas no recebimento das doses houve aumento da chance de hospitalização em UTI por patologia/sintomatologia respiratória. Um aspecto positivo do estudo decorre do fato de ser a primeira avaliação brasileira sobre o programa de uso da imunoglobulina palivizumabe. Assim, as condições aqui apresentadas podem estar ocorrendo em outros estados brasileiros.

Um estudo anterior que teve por objetivo avaliar a implantação dos CRIEs em todo o território nacional, porém sem considerar os postos de aplicação, apontou a Região Sudeste como a que concentra os serviços de melhor estrutura 14.

De modo geral, na presente pesquisa a adequação apenas parcial ou inadequação do processo não teve a estrutura como fator limitante. Ainda assim, são cabíveis as seguintes melhorias estruturais gerais: climatização das salas de aplicação, uso da sala exclusivamente para aplicação de imunobiológicos, e provisão de medicamentos e materiais necessários em situação de choque.

A justificativa para tais demandas é técnica. A climatização, com o uso de aparelho de ar-condicionado quente ou frio, conforme a necessidade, é importante para minimizar os riscos indesejados de alteração da temperatura dos imunobiológicos 15; para que os procedimentos sejam realizados com a máxima segurança, visando à redução do risco de contaminação para a equipe e usuários, indica-se o uso exclusivo da sala para aplicação de imunobiológicos 12; a existência de material para uso em situação de urgência é condição que pode viabilizar o cuidado na vigência deste desfecho, especialmente grave pela vulnerabilidade das crianças elegíveis 16, e a vinculação ao programa de profissional técnico-administrativo otimiza o processo de trabalho da equipe e, consequentemente, o cuidado de enfermagem 17.

O fato de quatro postos terem estrutura parcialmente adequada indica a necessidade de outras ações além daquelas anteriormente apontadas, por vezes de fácil resolução em nível local, dependendo apenas de empenho individual para correção da falha (como a distribuição adequada do mobiliário, ausência de decoração e uso de compressas de álcool a 70\%). Adequação do piso e proteção contra a luz solar demandam solicitação à gestão superior.

Também, destaca-se que um desses quatro postos (P1) apresenta mais fragilidades relacionadas aos recursos humanos; outro (P11) tinha mais deficiência de recursos físicos e materiais, e os demais equilibravam as desconformidades entre recursos físicos, materiais e humanos. Deficiências de recursos humanos próprias dos postos incluíam a não presença de enfermeiro ou enfermeiro supervisor e de médico, aspectos que podem ser facilmente justificáveis: o Conselho Federal de Enfermagem não considera que o auxiliar ou técnico de enfermagem esteja habilitado para tal função 18 , havendo indicação de que a organização das atividades técnicas de enfermagem seja privativa do enfermeiro; ainda, o protocolo de uso da imunoglobulina é claro quanto à necessidade de vinculação de médico ao programa, inclusive durante a aplicação 10, visto que as crianças elegíveis são altamente vulneráveis e, assim, há possibilidade de intercorrências graves, como a parada cardiorrespiratória e, consequentemente, a necessidade de cuidados emergenciais, oportunos e apropriados 19.

Para contribuir com a redução nas falhas no recebimento da imunoglobulina, desconformidades da estrutura precisam ser corrigidas: a falta da imunoglobulina durante o período de sazonalidade, ocorrência relatada por um dos locais de aplicação, e a ausência de protocolo e de capacitação da equipe executora, situações que podem contribuir para que a oferta não ocorra como preconizado. A recomendação de manutenção do protocolo de forma impressa facilita o acesso à informação e, então, fornece suporte ao adequado manejo clínico, com sequência e estratégias previamente definidas 20. A existência de capacitação inicial é essencial para evitar a busca de qualificação de maneira informal, como valendo-se apenas da leitura das bulas ou mesmo dos representantes de laboratórios. Embora não haja padronização estadual sobre a capacitação dos profissionais para o desenvolvimento do programa e sua periodicidade, aponta-se a necessidade de implantação de ações de educação permanente, essencial para qualificar os profissionais envolvidos 21 inclusive no manejo de reações adversas e evitar divergências na condução do programa, especialmente quando se considera haver grande rotatividade de pessoal nos serviços de saúde. A melhoria desses aspectos estruturais dependerá de ação da coordenação estadual do programa.

No contexto do processo, mesmo para os CRIE, a situação é pior do que a observada quando considerada a estrutura, visto que três foram classificados como parcialmente adequados e um como inadequado. Situação ainda mais negativa foi observada entre os postos de aplicação: apenas três 
foram classificados como adequados, dois eram parcialmente adequados e seis inadequados. Dentre os classificados como inadequados, destaca-se o P11 onde pouco mais de dois terços das crianças tiveram quatro falhas de aplicação. Dentre os problemas identificados, estavam a pouca participação do médico e inadequações na conservação do imunobiológico.

Embora na análise de estrutura apenas três locais de aplicação tenham apontado a falta do médico, a participação deste profissional no processo de desenvolvimento do programa foi menor, visto que em nove postos o médico não realizava o preconizado pelo Ministério da Saúde: orientação, prescrição e supervisão da aplicação 10.

A maioria dos locais de aplicação não mantinha a imunoglobulina conservada entre +2 e $+8^{\circ} \mathrm{C}$ após a diluição, apesar da recomendação do Ministério da Saúde relativa aos princípios da rede de frio, para que se evite o comprometimento da potência imunogênica do imunobiológico 12.

Três desconformidades, porém, são especialmente relevantes por guardarem relação direta com as falhas no recebimento da imunoglobulina: a não aplicação deste imunobiológico a crianças hospitalizadas, a não aplicação no mês anterior à sazonalidade e a ausência de busca ativa de faltosos, condição ainda mais preocupante quando se observa ter ocorrido em um CRIE.

Desde 2013, o Ministério da Saúde 10 indicou profilaxia às crianças internadas no período de sazonalidade do VSR, porém, a maioria dos locais de aplicação não viabiliza seu cumprimento, indicando a necessidade de incorporação desta recomendação. Esse fato pode ter sido potencializado pela proximidade entre a publicação da Portaria e o início do período sazonal e, consequentemente, da inclusão das crianças no programa.

Em cinco postos de aplicação a primeira dose não foi aplicada um mês antes da sazonalidade 10 . Destaca-se que o documento relativo à sazonalidade, do ano de 2015, indicava que em março daquele ano já havia circulação viral e, assim, falhas nessa prática podem prejudicar a prevenção 22 . A ausência da aplicação da imunoglobulina, quer seja pela falta do imunobiológico, quer pela ausência de busca ativa por parte do serviço ou ainda pela não aplicação da primeira dose no mês que antecede a sazonalidade pode causar prejuízos às crianças-alo dessa intervenção. Essa última desconformidade, que ocorreu em aproximadamente $85 \%$ das crianças participantes, pode também decorrer da falta de agilidade na obtenção do imunobiológico, bem como da autorização para seu uso, visto que apenas um CRIE e um posto podem autorizar a aplicação, cabendo aos demais encaminhar a ficha de solicitação ao Grupo de Vigilância Epidemiológica, que a encaminha ao Programa Estadual de Imunização para avaliação e posterior autorização.

A análise dos resultados do programa tomou por referência a necessidade de hospitalização em UTI por doença/sintomatologia respiratória. Na presente pesquisa, deixar de receber pelo menos uma vez o imunobiológico durante o período de sazonalidade do VSR não resultou em aumento de hospitalização. Porém, na medida em que aumentou a quantidade de falhas, aumentou a chance de hospitalização: a média de aumento por falha foi de 30\% ( $\mathrm{p}=0,003$; OR = 1,30; IC95\%: 1,09-1,55).

Nesse sentido, uma meta-análise realizada em 2009 apontou que a profilaxia resulta em redução de hospitalização em UTI em relação ao placebo ( $p=0,0007$; RR = 0,29; IC95\%: 0,14-0,59) 23. Outros estudos apontam vantagens do uso da palivizumabe entre crianças de risco, sendo a imunoglobulina considerada eficiente na profilaxia de hospitalização decorrente do VSR 23,24,25. Outra forma de medir o impacto das falhas: verificou-se que deixar de receber o imunobiológico nos meses de março, abril e junho resultou em hospitalização nos meses subsequentes (abril, maio e julho, respectivamente). Nesse sentido, outros estudos serão necessários para explicar tais achados.

Nesta pesquisa, o percentual de hospitalização em UTI relacionada à etiologia respiratória foi de $18,8 \%$, tanto para crianças que receberam todas as doses quanto para aquelas que perderam uma dose da imunoglobulina - praticamente o dobro do valor encontrado na coorte brasileira de 198 crianças, na qual a taxa foi de 9,1\% 25. O estudo IMpact 11, ensaio clínico que fundamentou a adoção da palivizumabe nos Estados Unidos, também encontrou taxa de hospitalização por VSR inferior a deste estudo: 10,6\% no grupo sem profilaxia e de 4,8\% no grupo que recebeu a imunoglobulina, o que correspondeu à redução de $55 \%$.

Outro estudo americano 26 teve por foco situações em que há imunização parcial, em decorrência de falha no procedimento de aplicação: é o caso de coorte retrospectiva realizada entre 2003 e 2009, com 8.443 crianças de alto risco, sendo que dois terços delas receberam profilaxia apenas parcial. 
Nesse caso, para crianças com imunização completa, os autores relataram taxa de hospitalização relacionada ao VSR de 7,9\%, maior do que aquela relatada no estudo IMpact $(4,8 \%) 11$.

A taxa de hospitalização obtida na presente investigação foi superior à de todos os estudos citados, o que pode decorrer da não realização de exame para isolar o agente infeccioso, ou seja, não foi confirmada a presença do VSR, podendo haver superestimação da taxa, já que em parte dos casos haverá outros agentes. Essa situação constitui a principal limitação deste estudo. Outro aspecto merece ser considerado: a coleta de dados sobre estrutura e processo ocorreu por telefone, o que pode ter resultado em subidentificação de desconformidades.

\section{Conclusão}

A estrutura do programa de uso da imunoglobulina palivizumabe no Estado de São Paulo é, em geral, favorável, mas investimentos ainda são necessários para qualificar os locais de aplicação e reduzir problemas que têm impacto mais direto nas falhas identificadas, como a oferta ininterrupta da imunoglobulina durante o período de sazonalidade, a disponibilização de protocolo e a capacitação da equipe executora periodicamente.

O processo de atenção do programa foi menos favorável. Nesse componente da avaliação, apenas três postos de aplicação foram considerados adequados e as melhorias devem ocorrer, principalmente, na busca ativa de faltosos, aplicação a crianças hospitalizadas e início da aplicação antes do período de sazonalidade, aspectos também diretamente relacionados às falhas no recebimento da imunoglobulina.

Quanto ao resultado, aponta-se a necessidade de respeitar a aplicação do número de doses preconizado, visto que há um aumento médio de $30 \%$ na chance de internação por doença/sintoma respiratório a cada falha. Ações nessa direção incluem evidenciar aos profissionais de saúde e às famílias a importância de se aplicar o imunológico com aprazamento indicado, não superior a trinta dias, e aprimorar o processo de atenção.

\section{Colaboradores}

Todos os autores participaram de todas as etapas de elaboração do artigo.

\section{Agradecimentos}

A Anna Ferrari, Érica Cardozo, Flávia Seuller, Larissa Funatsu, Mariana Dias, Marina Moraes, Maysa Gayoso e Raissa Amaro pelo auxílio na coleta de dados.

\section{Referências}

1. Cintra OAL, Owa MA, Machado AA, Cervi MC, Figueiredo LTM, Rocha GM, et al. Occurrence and severity of infections caused by subgroup A and B respiratory syncytial virus in children in southeast. Brazil J Med Virol 2001; 65:408-12.

2. Nascimento JP, Siqueira MM, Sutmoller F, Krawczuk MM, Farias V, Ferreira V, et al. Longitudinal study of acute respiratory diseases in Rio de Janeiro: occurrence of respiratory viruses during four consecutive years. Rev Inst Med Trop São Paulo 1991; 33:287-96.

3. Stralioto SM, Nestor SM, Siqueira MM. Respiratory syncytial virus groups A and B in Porto Alegre, Brazil, from 1990 to 1995 and 1998. Mem Inst Oswaldo Cruz 2001; 96:155-8.

4. Nair H, Nokes DJ, Gessner BD, Dherani M, Madhi SA, Singleton RJ, et al. Global burden of acute lower respiratory infections due to respiratory syncytial virus in young children: a systematic review and meta-analysis. Lancet 2010; 375:1545-55. 
5. Hall CB, Weinberg GA, Iwane MK, Blumkin AK, Edwards KM, Staat MA, et al. The burden of respiratory syncytial virus infection in young children. N Engl J Med 2009; 360: 588-98.

6. Simões EAF. Immunoprophylaxis of respiratory syncytial virus: global experience. Respir Res 2002; 3 Suppl 1:S26-33.

7. Salomão Junior JB, Gardinassi LGA, Simas PVM, Bittar CO, Souza FP, Rahal P, et al. Human respiratory syncytial virus in children hospitalized for acute lower respiratory infection. J Pediatr (Rio J.) 2011; 87:219-24.

8. Riccetto AGL, Ribeiro JD, Silva MTN, Almeida RS, Arns CW, Baracat ECE. Respiratory syncytial virus (RSV) in infants hospitalized for acute lower respiratory tract disease: incidence and associated risks. Braz J Infect Dis 2006; 10:357-61.

9. Secretaria de Estado da Saúde de São Paulo. Resolução SS no 249, de 13 de julho de 2007. Aprova Norma Técnica elaborada pela Coordenadoria de Controle de Doenças - Centro de Vigilância Epidemiológica e Coordenadoria de Ciência, Tecnologia e Insumos Estratégicos de Saúde, que estabelece as diretrizes para a prevenção da infecção pelo Vírus Sincicial Respiratório - VSR no âmbito do Sistema Único de Saúde do Estado de São Paulo. Diário Oficial do Estado de São Paulo 2007; 14 jul.

10. Ministério da Saúde. Portaria no 522, de 13 de maio de 2013. Aprova o protocolo de uso do palivizumabe. Diário Oficial da União 2013; 14 mai.

11. Suresh G. Impact-RSV study group report. Pediatrics 1999; 104:993-4.

12. Departamento de Vigilância das Doenças Transmissíveis, Secretaria de Vigilância em Saúde, Ministério da Saúde. Manual de normas e procedimentos para vacinação. Brasília: Ministério da Saúde; 2014.

13. Donabedian A. Basic approaches to assessment: structure, process and outcome. In: Donabedian A, editor. Explorations in quality assessment and monitoring: the criteria and standards of quality. v. 1. Ann Arbor: Health Adiministration Press; 1980. p. 77-125.

14. 'Nóbrega LAL, Novaes HMD, Sartori AMC. Avaliação da implantação dos Centros de Referência para Imunobiológicos Especiais. Rev Saúde Pública 2016; 50:58.

15. 'Departamento de Vigilância Epidemiológica, Secretaria de Vigilância em Saúde, Ministério da Saúde. Manual de rede de frio. 4a Ed. Brasília: Ministério da Saúde; 2013.

16. Bernd AG, Sole D, Pastorino AC, Prado EA, Castro FFM, Rizzo MCV, et al. Anafilaxia: guia prático para o manejo. Arquivos de Asma, Alergia e Imunologia 2006; 29:283-91.

17. Pai DD, Lautert L. O trabalho em urgência e emergência e a relação com a saúde das profissionais de enfermagem. Rev Latinoam Enferm 2008; 16:439-44.
18. Conselho Federal de Enfermagem. Decreto no 94.406, de 8 de junho de 1987. Regulamenta a Lei no 7.498, de 25 de junho de 1986, que dispõe sobre o exercício da Enfermagem, e dá outras providências. Diário Oficial da União 1987; 9 jun.

19. Gonzalez MM, Timerman S, Oliveira RG, Polastri TF, Dallan LAP, Araújo S, et al. I diretriz de ressuscitação cardiopulmonar e cuidados cardiovasculares de emergência da Sociedade Brasileira de Cardiologia: resumo executivo. Arq Bras Cardiol 2013; 100:105-13.

20. Polanczyk CA, Prompt CA, Ferreira J, Caye L, Vacaro R, Kuchenbecker R, et al. Protocolos assistenciais como estratégia de adesão às melhores práticas clínicas e otimização de recursos. Brasília: Instituto de Pesquisa Econômia Aplicada; 2015.

21. Oliveira FMCSN, Ferreira EC, Rufino NA, Santos MSS. Educação permanente e qualidade da assistência à saúde: aprendizagem significativa no trabalho da enfermagem. Aquichan 2011; 11:48-65.

22. Ministério da Saúde. Nota técnica conjunta no 05/2015 - CGSCAM/DAPES/SAS/MS, CGAFME/DAF/SCTIE/MS e CGDT/DEVIT/SVS/ MS. Estabelecer a sazonalidade do vírus sincicial respiratório no Brasil e oferecer esclarecimentos referentes ao protocolo de uso do palivizumabe. Brasília: Ministério da Saúde; 2015.

23. Morris SK, Dzolganovski B, Beyene J, Sung L. A meta-analysis of the effect of antibody therapy for the prevention of severe respiratory syncytial virus infection. BMC Infect Dis 2009; 9:106.

24. Feltes TF, Cabalka AK, Meissner C, Piazza FM, Carlin DA, Top FH, et al. Palivizumab prophylaxis reduces hospitalization due to respiratory syncytial virus in young children with hemodynamically significant congenital heart disease. J Pediatr 2003; 143:532-40.

25. Monteiro AIMP, Bellei NCJ, Sousa AR, Santos AMN, Weckx LY. Respiratory infections in children up to two years of age on prophylaxis with palivizumab. Rev Paul Pediatr 2014; 32:152-8.

26. Krilov LR, Masaquel AS, Weiner LB, Smith DM, Wade SW, Mahadevia PJ. Partial palivizumab prophylaxis and increased risk of hospitalization due to respiratory syncytial virus in a Medicaid population: a retrospective cohort analysis. BMC Pediatr 2014; 14:261. 
Abstract

This study aimed to assess the program for use of the monoclonal antibody palivizumab in São Paulo State, Brazil. The evaluation adopted the frame of reference proposed by Donabedian, and the data were discussed on the basis of the guidelines from the Ruling on the use of palivizumab in the national network and in the Manual on Standards and Procedures for Vaccination. Sixteen application services in the state were included, with 693 children/mothers enrolled in the program in 2014 (85.1\% of the eligible population). For the structure and process evaluation, scores were created that allowed classifying the application services as adequate, partially adequate, and inadequate (non-compliance rates were $\leq 10 \%, 11$ $20 \%$, and $>20 \%$, respectively). Results were evaluated according to the association between failure to administer palivizumab and need for hospitalization due to respiratory disease/symptoms, based on the point and interval odds ratios, with 95\% confidence interval and critical p-value $<0.05$. Of the 11 application services whose structure was classified as adequate, only two showed adequate process, four showed inadequate process, and five partially adequate process. Risk of ICU admission due to respiratory disease/symptoms increased on average by 30\% for each failure ( $p=0.003$; $O R=1.30$; 95\%CI: 1.09-1.55). In conclusion, having a favorable structure for the program for use of palivizumab in São Paulo State did not necessarily result in an adequate process. In general, the situation with the structure was better than the process. All doses of the monoclonal antibody need to be administered in order to prevent hospitalization from respiratory disease/symptoms.

Palivizumab; Immunoglobulins; Passive Immunization; Risk Groups; Program Evaluation

\section{Resumen}

El objetivo de este estudio fue evaluar el programa de uso de la inmunoglobulina palivizumab en el Estado de São Paulo, Brasil. Se adoptó el sistema referencial de evaluación propuesto por Donabedian, donde los datos se discutieron en base a las recomendaciones del decreto que regula el uso del palivizumab en la red nacional y en el Manual de Normas y Procedimientos para la Vacunación. Se incluyeron 16 espacios de aplicación en el estado, así como a 693 niños/madres inscritas en el programa en 2014 (85, 1\% de la población elegible). Para la evaluación de la estructura y proceso se crearon marcadores que permitieron clasificar los lugares de aplicación como: adecuado, parcialmente adecuado e inadecuado, cuando había hasta un 10\%, de 11-20\% y superior al 20\% de discordancias, respectivamente. Para la evaluación del resultado, se buscó la asociación entre el fallo en la toma del palivizumab y la necesidad de hospitalización por enfermedad/sintomatología respiratoria, basándose en la razón de probabilidades puntual y con intervalos, con un intervalo del $95 \%$ de confianza y valor de p crítico $<0,05$. De los 11 puestos de aplicación que contaron con una estructura clasificada como adecuada, solamente dos presentaron un proceso adecuado, cuatro presentaron proceso inadecuado y cinco parcialmente adecuados. El riesgo de hospitalización en la UTI por enfermedad/sintomatología respiratoria aumentó de media un $30 \%$ con cada fallo $(p=0,003$; $O R=1,30$; IC95\%: 1,09-1,55). Se concluye que contar con una estructura del programa de uso de la inmunoglobulina en el Estado de São Paulo no resultó favorable, necesariamente, en un proceso adecuado. En general, la situación de la estructura fue mejor que la del proceso. Se mostró relevante la aplicación de todas las dosis de inmunoglobulina para la prevención del internamiento por enfermedad/sintomatología respiratoria.

Palivizumab; Inmunoglobulinas; Inmunización Pasiva; Grupos de Riesgo; Evaluación de

Programas y Servicios de Salud
Recebido em 06/Jul/2016

Versão final reapresentada em 09/Nov/2017

Aprovado em 12/Jan/2018 